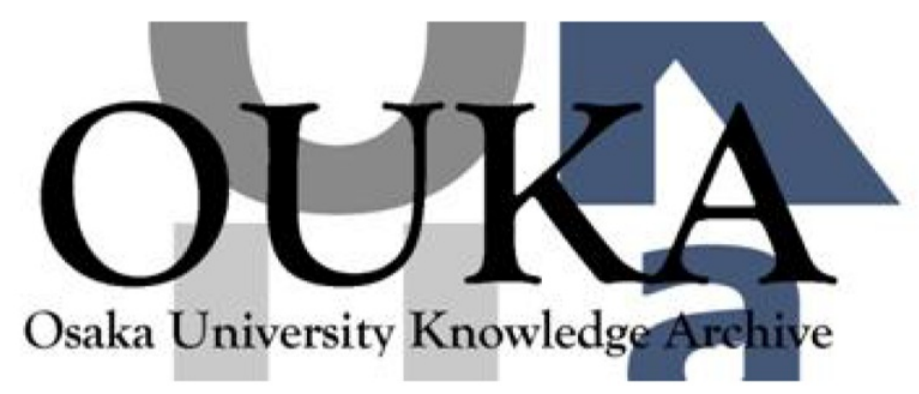

\begin{tabular}{|c|l|}
\hline Title & $\begin{array}{l}\text { Iterative processing on hybrid optical parallel } \\
\text { array logic system with selectable coherent } \\
\text { correlator }\end{array}$ \\
\hline Author(s) & $\begin{array}{l}\text { Miyazaki, Daisuke; Kakizaki, Sunao; Tanida, Jun } \\
\text { et al. }\end{array}$ \\
\hline Citation & Applied Optics. 32(17) p.3053-p.3058 \\
\hline Issue Date & 1993-06-10 \\
\hline oaire:version & VoR \\
\hline URL & https://hdl.handle. net/11094/3411 \\
\hline rights & \\
\hline Note & \\
\hline
\end{tabular}

Osaka University Knowledge Archive : OUKA

https://ir. Library. osaka-u. ac. jp/

Osaka University 


\title{
Iterative processing on a hybrid optical parallel array logic system with a selectable coherent correlator
}

\author{
Daisuke Miyazaki, Sunao Kakizaki, Tsuyoshi Konishi, Jun Tanida, and Yoshiki Ichioka
}

\begin{abstract}
A new version of an experimental system of an optoelectronic hybrid optical parallel array logic system is constructed. The experimental system is composed of a two-dimensional correlator with selectable holographic filters and parallel electronic circuits. The system can process images consisting of $3 \times 3$ pixels at a time. Several types of processing are achieved both without and with dynamic selection of the holographic filters. On the experimental system, parallel operations of several logical functions and data transmission are demonstrated. Processing rates of iterative operation without and with filter selection are 278 and 143 frames/s, respectively.
\end{abstract}

Key words: Digital-optical computing system, holographic filter.

\section{Introduction}

A digital-optical computing system is expected to be an excellent information-processing system with high computing capability from the features of light propagation such as high speed, parallelism, and cross-talkfree interconnection. We have been studying the optical parallel array logic system (OPALS) ${ }^{1,2}$ as an instance of a highly parallel optical-digital processing system. The OPALS is designed on the basis of the parallel computing principle called optical array logic $(\mathrm{OAL})^{2,3}$ OAL is based on spatial encoding and two-dimensional (2-D) correlation; it is capable of executing various types of parallel processing for $2-\mathrm{D}$ images and makes use of the advantages of light propagation.

Two types of preliminary optoelectronic hybrid systems have been constructed to verify operations of the OPALS.,5 The first system is composed of a multichannel reflective correlator with a 2-D galvanometer mirror, which can process $20 \times 20$ pixels at a time. ${ }^{4}$ In this system, however, some parts of the process are executed by a personal computer; also, a liquid-crystal display and a CCD camera are used for

When this work was performed, the authors were with the Department of Applied Physics, Faculty of Engineering, 2-1 Yamadaoka, Suita, Osaka 565, Japan. S. Kakizaki is now with Hitachi Ltd., Central Research Laboratory, 1-280, Higashi-koigakubo, Kokubunji-shi, Tokyo 185, Japan.

Received 24 February 1992.

0003-6935/93/173053-06\$06.00/0.

(c) 1993 Optical Society of America. displaying and detecting images, respectively. Therefore the capability of parallel processing, which is an advantage of the OPALS, cannot be exhibited owing to serial processing in the electronics part. In order to demonstrate complete parallelism of the OPALS, it is important to construct such a system with parallel electronics even though the pixel number is rather small. For this purpose the second system was constructed with a correlator based on a multiplepinhole-camera system and parallel electronic circuits, which can process the signals for all pixels simultaneously. ${ }^{5}$ Although this correlator has a simple configuration, it processes the input images with a low density of pixels and has poor scalability. Since the capability of a correlator in the OPALS determines the performance and the flexibility of an OAL processor, ${ }^{3}$ we must use a more powerful correlator. To do so, we adopt a coherent correlator with holographic filters, ${ }^{6}$ which has the advantages of processing capability and flexibility as compared with previous correlators.

Several methods of applying the holographic filters to optical-digital computing systems have been pre-

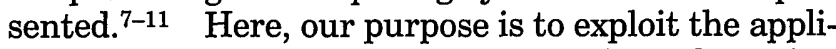
cability and usefulness of the correlator by using holographic filters in the OPALS. Subjects that we examine include the feasibility of holographic filters, the capability of optoelectronic interfaces, effects of coherent noise, reliability of the optical system, usefulness of a spatial light modulator (SLM), necessity of the parallel electronic circuits, and consideration toward development of the improved version of meth- 
ods. We construct the hybrid OPALS (H-OPALS) with a selectable coherent correlator and fully parallel electronic circuits, and we examine the performance of the system. In Section 2, we explain briefly OAL and the OPALS. In Section 3, we describe specifications of the constructed system. In Section 4 we demonstrated several experimental results executed on the system. In Section 5, we discuss requirements to improve the performance of the H-OPALS.

\section{Optical Array Logic and the Optical Parallel Array Logic System}

$\mathrm{OAL}$ is a technique for implementing arbitrary neighborhood operations for 2-D images in parallel. 2,3 Figure 1 shows the processing procedures of an OAL. Two binary input images $\mathrm{A}$ and $\mathrm{B}$ are converted into a single coded image with the coding rule shown in Fig. 1. The coded image is correlated separately with different sets of delta functions, called operation kernels. Individual correlated images are sampled spatially at one-pixel intervals along the vertical and horizontal directions. Assigning dark cells to logical 1 and bright cells to logical 0 (dark true logic), we compute the product terms of neighborhood operation. For an arbitrary operation a logical sum is applied to a set of sampled images, and then logical inversion is executed to convert the dark true logic into bright true logic. The operation of OAL is specified by a combination of operation kernels used for 2-D correlation. Procedures implementing OAL are simple and suitable for optical processing, so that parallelism of optics can be used for parallel digital processing.

The OPALS is a conceptual system that executes OAL effectively ${ }^{1,2}$; it is composed of a parallel processing unit that executes OAL, an internal parallel feedback line, and parallel input-output ports. The feedback line connects the output of the OAL processor to one of the inputs for effective iterative operation. The H-OPALS is a version of the system that can be constructed by commercially available optoelectronic devices. Thus we choose the H-OPALS as a framework for the experimental system.

\section{Experimental Hybrid Optical Parallel Array Logic System}

\section{A. System Overview}

We describe the construction of a new version of the H-OPALS. To construct the H-OPALS executing

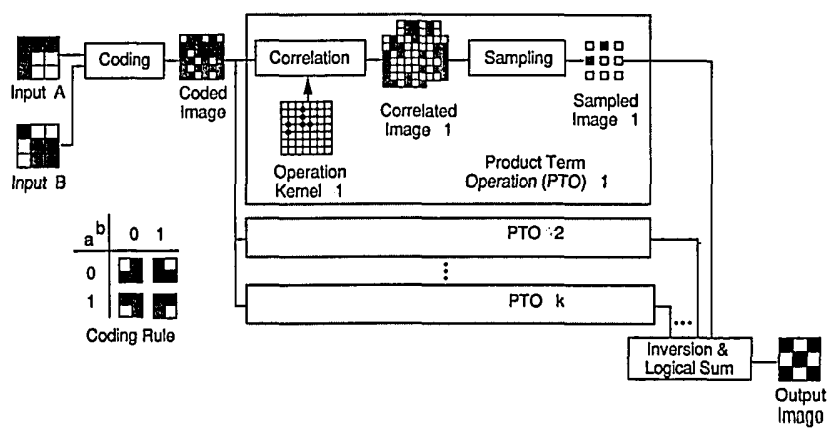

Fig. 1. Procedures of optical array logic.
$\mathrm{OAL}$, we must develop the following three functional subsystems: an encoder, a 2-D correlator, and a decoder. We designed the experimental system so that encoding and decoding are implemented electronically, whereas 2-D correlation is executed optically in parallel. In the developed system the functions of memory and nonlinear processing required in encoding and decoding are achieved with electronics rather than optics because parallel data communication in both the encoder and the decoder is based on pixel-topixel transmission. This interconnection is much simpler than that in a 2-D correlator. Structures of the encoder and the decoder based on pixel-to-pixel interconnections are suitable for large-scale integration because of the simplicity and the individuality of the processing at every pixel.

Figure 2 shows a block diagram of the developed H-OPALS. Both encoder and decoder consist of parallel electronic circuits, a light-emitting-diode (LED) array, and a photodetector (PD) array. The correlator is composed of an optically addressed SLM, an optical-shutter array, and a holographic correlation-filter array. The optically accessed SLM can access every pixel simultaneously. A coded image is displayed on the LED array of the encoder, projected onto the SLM, and converted into a coherent image. The coherent coded image is correlated with a combination of correlation filters recorded as Fourier holograms of operation kernels. A desired filter is selected by opening one array of optical shutters in front of the filter array. The correlated image is detected by the PD array and converted into electric signals for electronic processing in the decoder. The decoder executes the spatial sampling, logical inversion, and logical-sum operations.

The resultant image is displayed on the LED array and fed back to the encoder through the optical feedback path for iterative operation. In this case the feedback line is implemented by a simple imaging system, which can be replaced by electronic wiring. However, we consider the modularized version of the

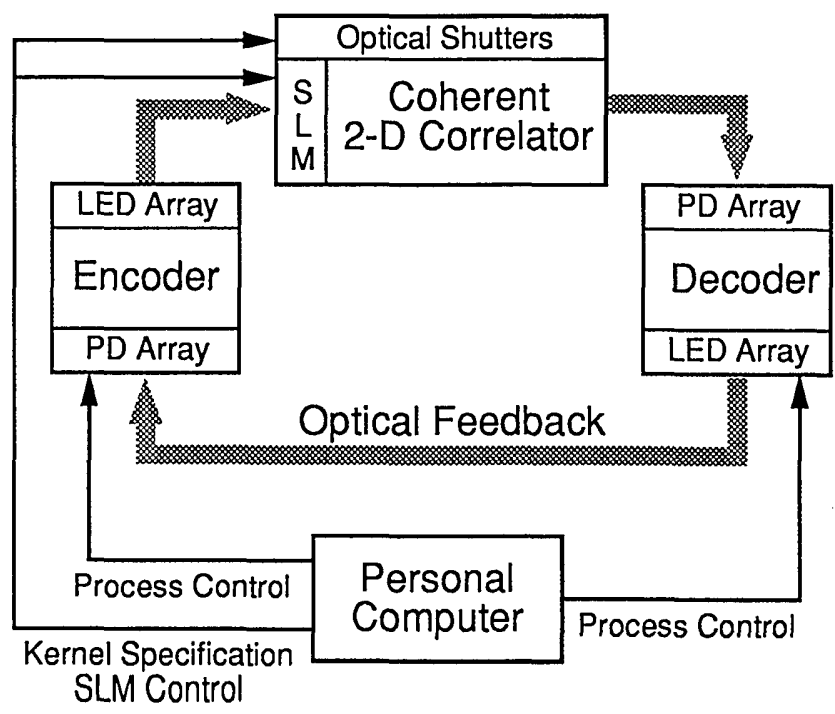

Fig. 2. Block diagram of the constructed H-OPALS. 
OPALS, which is composed of separable functional modules (the encoder, the correlator, the decoder, and so on) that are linked optically. ${ }^{12}$ To achieve such a modularized system, we choose to keep the feedback line optical. This permits us the flexibility of inserting several types of modules or optical systems as needed.

All operations and all data transmission in the system are achieved in parallel for every pixel. A sequence of system operations is controlled by a personal computer.

In the experimental system, parallel processing circuits for $3 \times 3$ pixels are constructed individually. This small pixel number is not attractive for parallel processing; however, the experimental system provides important and fundamental information useful for the construction of a future massively parallel optoelectronic-processing system. A small-scale parallel system can be constructed rather easily with available devices and technologies, and it can verify the system operation and examine the feasibility of optical systems or devices required for parallel optical processing. This system has the minimum number of pixels needed for neighborhood operation. Of course, the space-bandwidth product of the optical system is much more than $3 \times 3$. The electronics for the H-OPALS consists of circuits operating independently for every pixel. If we desire to treat data consisting of larger pixel numbers in the future, it is possible to integrate electronic circuits using largescale-integration techniques.

\section{B. Selective Two-Dimensional Coherent Correlator}

Figure 3 shows the coherent 2-D correlator used in the experimental system. The correlator is equvialent to a modified two-focal-length optical correlator, ${ }^{13}$ in which the collimator lenses required in the classical $4 f$ correlator are eliminated and the variablescale property of the Fourier transform is available. The diffuser and the filter array are set on the conjugate planes of lens L1, and the SLM and the PD array are on those of lens L2. Lens L1 provides the Fourier transform of the input image on the filter plane with a quadratic phase factor. This phase factor is compensated by lens L2. Lens L2 also executes a Fourier transform of the transmitted image through a correlation filter, and the result is detected by the PD array. Although another quadratic phase factor is introduced on the PD plane, it does not affect the result detected by intensity.

To convert a coded image displayed on the LED array into a coherent one, we use a light-addressed photoconductor and a smectic $C^{*}$ liquid-crystal SLM (LAPS-SLM) produced by Seiko Instruments, Inc., ${ }^{14}$ in the experimental system. This device uses ferroelectric liquid crystals (FLC's) for modulating the state of polarization of the read light. Using a polarizer or a polarizing beam splitter (PBS), we can modulate the amplitude of the light. The resolving power of the LAPS-SLM is more than 100 line pairs $/ \mathrm{mm}$, and its typical contrast ratio is $150: 1$. This device can operate at a frame rate of $1.5 \mathrm{kHz}$.

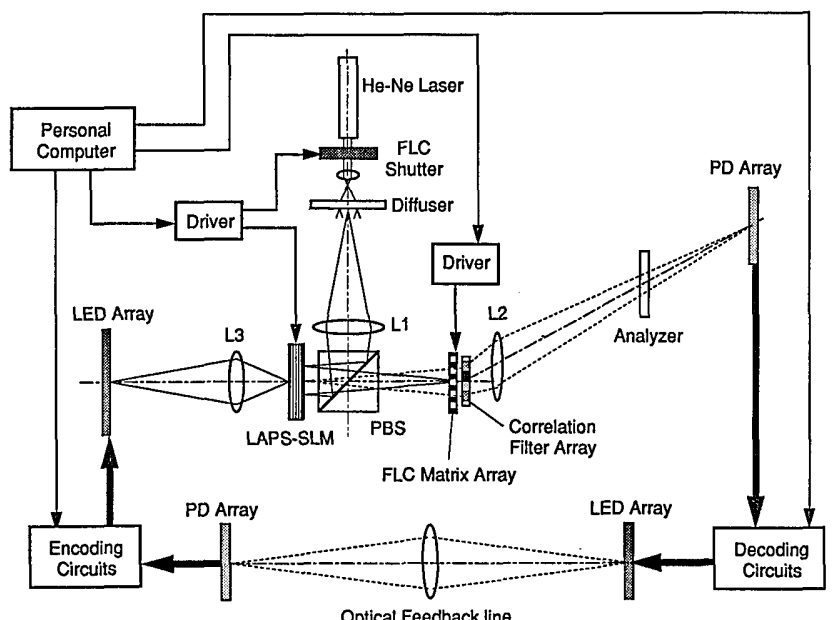

(a)

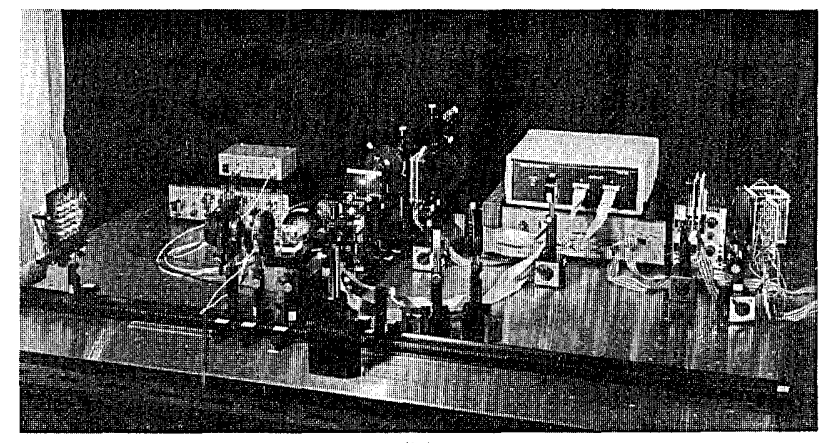

(b)

Fig. 3. Two-dimensional coherent correlator with a holographic filter array: (a) schematic diagram, (b) photograph of the system.

The LAPS-SLM used in the system has no reflective mirror between the liquid crystal and the $\alpha-\mathrm{Si}: \mathrm{H}$ photoconductor layers. Thus, to avoid overwrite by the read light, we must set an optical shutter to block the read light during the write cycles of the SLM.

Several operation kernels for different operations are recorded as Fourier holograms created from assemblies of delta functions, which are arranged as a correlation-filter array. When we fabricate a Fourier hologram of an operation kernel, a point consisting of the operation kernel is selected and recorded as a Fourier transform hologram. Then, the other points are recorded sequentially in the same manner upon the common hologram. The reason why we use such a multiple-exposure technique is to eliminate undesired noise generated by interference among the recorded points themselves. For example, to make a holographic filter for an AND operation composed of three delta functions, the exposure process is repeated three times.

In the experiments, two types of holographic plate, an Agfa Gevaert 10E75 plate and a Newport thermoplastic plate, are used for recording correlation filters. The filter recorded on the $10 \mathrm{E} 75$ plate is bleached to increase its diffraction efficiency. We made the holographic filters with 10 exposures. For the 10E75, $12 \%$ of diffraction efficiency is obtained, and for the thermoplastic plate, $15 \%$ is obtained. 
To achieve dynamic operation of the system, we need a mechanism for selecting specific correlation filters in a filter array. For this purpose an opticalshutter array is set in front of the correlation-filter array. The optical-shutter array used is a $6 \times 6 \mathrm{FLC}$ matrix array, produced by Displaytech, Inc., that is capable of controlling all shutters in parallel. This device serves to display several prestored images sequentially at a frame rate of up to $10 \mathrm{kHz}$. A diffuser is inserted to spread the light beam from a $30-\mathrm{mW} \mathrm{He}-\mathrm{Ne}$ laser over the filter array. Selection of a filter is achieved by opening shutters. Either one or multiple filters can be selected at a time, which increases the flexibility of the system operation. Although speckle noise caused by the diffuser is observed on the correlated image, it is irrelevant if the fine structure of the speckle noise is much smaller than the pixel structure of the correlated image.

\section{Electronic-Processing Components}

In the experimental system, nine individual electronic circuits are constructed to process images with $3 \times 3$ pixels in parallel. In both the encoder and the decoder, Toshiba's red LED's (TLRA130-C's) are used to display images. Toshiba phototransistors (TPS613's) are used to detect the optically processed images. Most of the processing circuits are made of transistor-transistor-logic integrated circuits (TTLIC's). Encoding circuits for one pixel consist of several TTL-IC's: an input selector for electric and optical inputs, two D-type flip-flops to latch data, and a two-to-four decoder. To display the coded image, we assign $2 \times 2$ LED's to every pixel, and one of them emits light according to the coding rule. Decoding circuits consist of JK-type flip-flops for sequential oR operation. They are controlled by a personal computer, an Epson PC-286VS, through a parallel inputoutput port.

\section{Experimental Results of the Hybrid Optical Parallel Array Logic System}

We execute three types of parallel processing on the constructed H-OPALS. First, simple parallel logical functions are implemented to verify basic operation of the system. Second, to demonstrate the capability of fast iterative processing of the constructed system, we attempt to execute inversion of an image repeatedly. These operations are pixel-by-pixel processing, and hence a single correlation filter is used without selecting filters dynamically. Third, to verify the neighborhood operation, by selecting filters with the optical-shutter array, we demonstrate repetitive parallel data transmission in four directions.

\section{A. Parallel Logical Operations}

The 16 logical functions of two binary variables are basic and important operations in digital computing. Figure 4 shows experimental results for parallel AND, XOR, and OR operations. Two input images $A$ and $B$, shown in Fig. 4(a), are encoded into a coded version by the encoder, as shown in Fig. 4(b). The coded image
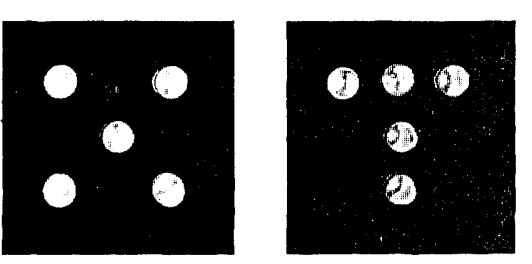

(a)
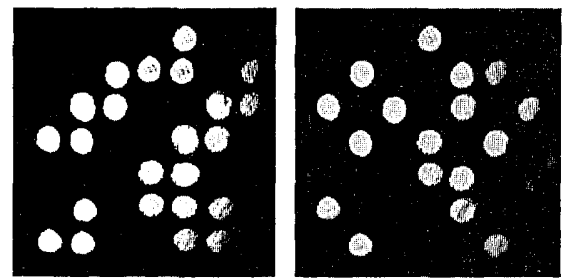

(c)
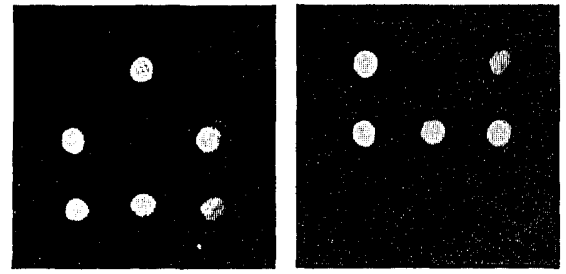

(d)

Fig. 4. Experimental results of parallel logic operations: (a) input images A and B; (b) coded image; (c) correlated images A AND $B, A$ xor B, A or B; (d) sampled images A AND B, A xor B, A or B.

is correlated with different filters, as shown in Fig. 4(c). Outputs of parallel AND, XOR, and OR operations are as shown in Fig. 4(d). Correct results are obtained by dark true logic according to the procedure of the OAL.

\section{B. Iterative Image Inversion}

As an example of simple iterative processing, inversion of an image is executed repeatedly. In this operation, individual pixel values in the image can be changed alternately with one operation kernel. Figure 5 shows the signal waveforms of (1) supplied voltages for the SLM, (2) the intensity of the read light, (3) the response of a $\mathrm{PD}$, and (4) the status of the corresponding pixel's value. In the signal for the

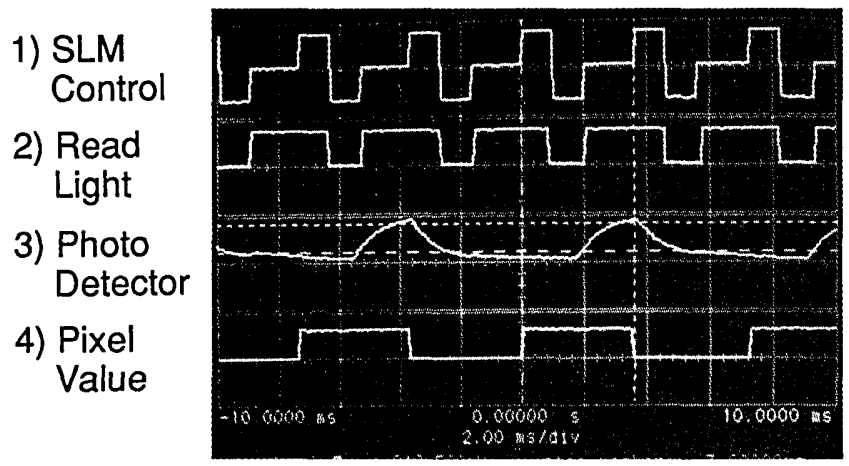

Fig. 5. Experimental results of repetitive image inversion: the waveforms, starting from the top, are for SLM control, the read light, the response of a $\mathrm{PD}$, and the pixel value. 
SLM the periods of positive, negative, and zero voltages correspond to those of erasing, writing, and holding, respectively. The read light for the SLM is blocked during the write period by the optical shutter. From these waveforms it becomes clear that the experimental system drives at a rate of $\sim 278$ frames $/ \mathrm{s}$ without filter selection. This processing speed is restricted by the response time of the amplifier of photocurrent detected by the PD.

\section{Iterative Processing of Data Transmission}

Data transmission is an operation for transferring specific data to the desired direction on an image plane in parallel. This operation is attempted for achieving flexible-structured data processing. ${ }^{15}$ Processing itself can be implemented by parallel-shift operations.

Figure 6(c) shows the pattern of the operation kernel for cyclic-shift operation in the upper direction. The shaded square in the operation kernel indicates the origin of a neighborhood area. The signals at the uppermost pixels are shifted to the lowermost pixels. We made four types of holographic filter on a thermoplastic plate for cyclic-shift operations. They serve

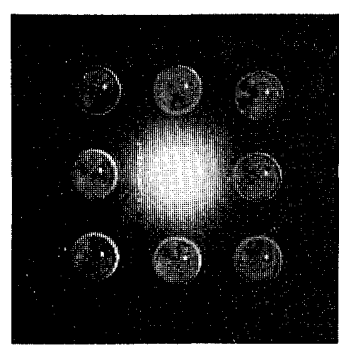

(a)

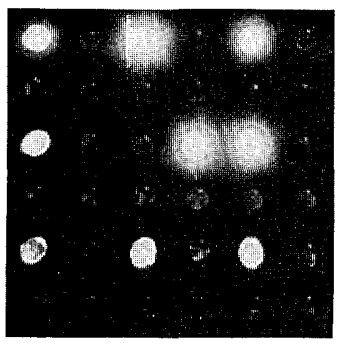

(b)

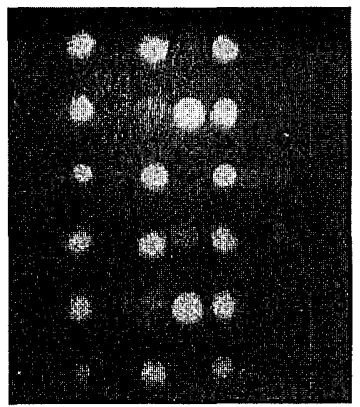

(d)

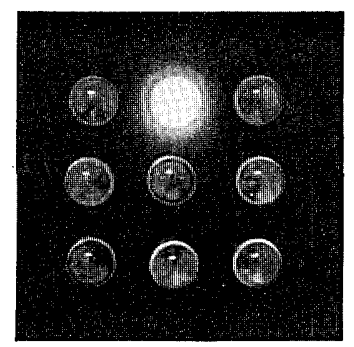

(e)

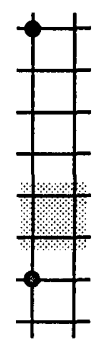

(c)

Fig. 6. Experimental results of shift operation in the upper direction: (a) input image; (b) coded image; (c) operation kernel pattern, in which the shaded square indicates the origin of a neighborhood area; (d) correlated image; (e) output image. to shift the image B to the upper, lower, right, and left directions by the amount of one pixel. The filter array is set in the experimental system, and the shutter array selects a filter to shift the image B along one of the four directions. Figure 6 shows the experimental results of a shift operation from the center to the upper direction. Figure 6(a) is the input image $B$ that has a data point located at the center pixel. Since the input image A does not affect the operation, all pixel values of the image $A$ are set to be zero. Figure $6(\mathrm{~b})$ is a coded image for the images $B$ and $A$. The coded image is displayed on the $6 \times 6$ LED array of the encoder. Figure 6(d) shows the image correlated with the operation kernel pattern shown in Fig. 6(c). Figure 6(e) shows the image sampled and inverted with the decoder (the final results). It can be seen that the correct results are obtained.

The iterative processing of the shift operation with selection of one of the four filters by the shutter array can be executed at the rate of $\sim 143$ frames/s. The processing speed is also restricted by the response time of the amplifier of photocurrent detected by the PD.

\section{Discussion}

As pointed out in Section 4, the processing speed of the experimental system is restricted mainly by the response time of the amplifier of photocurrent detected by the PD's. The constructed optical system consumes a large amount of light power owing to the low power efficiency of the SLM and the low diffraction efficiency of the correlation filter. Because only $\sim 5 \%$ of the input light power reaches the PD, high amplification of the detected signal is needed. In addition, processing of data transmission needs more time than that for inversion because, for data transmission, four filters must be illuminated at a time, and hence the effective light signal detected by a single PD decreases. Therefore, in order to build a system with high performance, we need bright coherent light sources, an SLM with high reflective efficiency, and holographic filters with high diffraction efficiency.

The optical system used, however, has an inherent disadvantage in power usage. The system uses only one coherent light source for multiple filters. Moreover, the light power is divided to all pixels on the PD array. Therefore, optical power reaching a pixel on the PD array is decreased and is inversely proportional to the product of the number of filters and pixels.

One method for avoiding this problem involves constructing the system so that it uses a 2-D laser diode (LD) array as light sources. ${ }^{11}$ We can rapidly select the filter by emitting light from the corresponding LD instead of by opening an optical-shutter array. Since an LD is used for a specific filter, this method has higher efficiency for light power usage as compared with the method of dividing the light beam emitted from a single light source over all the filters. If we increase the number of LD's in this configura- 
tion, we can increase the number of filters without decreasing the light power to individual detectors.

An alternative configuration of a correlator is one that uses an LD array for displaying a coded image. ${ }^{16,17}$ The correlator is robust for spatial incoherence and has a compact configuration because we can eliminate an SLM for incoherent-to-coherent conversion. LD's corresponding to the oN-state pixels are emitted and diffracted by holographic filters. In this configuration, light power from an LD is divided into the number of dots in the operation kernel, which is usually smaller than the number of pixels. Therefore, efficiency of light power reaching the individual detectors is higher than that of the above two configurations. As a result, this correlator has high scalability.

The experimental system is constructed with separate optical and optoelectronic components. To increase the number of pixels to be processed and to improve reliability, we need to develop optoelectronic devices specially designed for the OPALS by making use of large-scale-optoelectronic-integration ${ }^{18}$ and integrated-optics techniques. ${ }^{19}$

The authors gratefully acknowledge Tadao Iwaki of Seiko Instruments, Inc., for providing us the chance to use the LAPS-SLM in the experimental system.

\section{References}

1. J. Tanida and Y. Ichioka, "OPALS: optical parallel array logic system," Appl. Opt. 25, 1565-1570 (1986).

2. J. Tanida and Y. Ichioka, "A paradigm for digital optical computing based on coded pattern processing," Intl. J. Opt. Comput. 1, 113-128 (1990).

3. J. Tanida, M. Fukui, and Y. Ichioka, "Programming of optical array logic. 2: Numerical data processing based on pattern logic," Appl. Opt. 27, 2931-2939 (1988).

4. J. Tanida, J. Nakagawa, E. Yagyu, M. Fukui, and Y. Ichioka, "Experimental verification of parallel processing on a hybrid optical parallel array logic system," Appl. Opt. 29, 2510-2521 (1990).

5. D. Miyazaki, J. Tanida, and Y. Ichioka, "Construction of modularized OPALS using optoelectronic devices," Jpn. J. Appl. Phys. 29, L1550-L1552 (1990).
6. S. Kakizaki, D. Miyazaki, E. Yoshikawa, J. Tanida, and Y. Ichioka, "Hybrid optical array logic system," in Optics for Computers: Architectures and Technologies, G. J. Lebreton, ed., Proc. Soc. Photo-Opt. Instrum. Eng. 1505, 199-205 (1991).

7. B. K. Jenkins, P. Chavel, R. Forchheimer, A. A. Sawchuck, and T. C. Strand, "Architectural implications of a digital optical computer," Appl. Opt. 23, 3465-3474 (1984).

8. J. Taboury, J. M. Wang, P. Chavel, and F. Devos, "Optical cellular processor architecutre. 2: Illustration and system considerations," Appl. Opt. 28, 3138-3147 (1989).

9. Y. Takaki and H. Ohzu, "Holographic function/interconnection module," Appl. Opt. 30, 2825-2832 (1991).

10. J. B. McManus, R. S. Putnam, and H. J. Caulfield, "Switched holograms for reconfigurable optical interconnection: demonstration of a prototype device," Appl. Opt. 27, 4244-4250 (1988).

11. D. P. Casasent and E. C. Botha, "Multifunctional optical processor based on symbolic substitution," Opt. Eng. 28, 425-433 (1989).

12. J. Tanida and Y. Ichioka, "Modular components for an optical array logic system," Appl. Opt. 26, 3954-3960 (1987).

13. K. H. Fielding, J. L. Horner, and C. K. Makekau, "Modified two-focal-length optical correlator," Appl. Opt. 29, 4332-4333 (1990).

14. S. Yamamoto, R. Sekura, J. Yamanaka, T. Ebihara, N. Kato, and H. Hoshi, "Optical pattern recognition with LAPS-SLM (1)/light addressed photoconductor and smectic $C^{*}$ liquid crystal spatial light modulator," in Computer and Optically Formed Holographic Optics, 1. Cindrich and S. H. Lee, eds., Proc. Soc. Photo-Opt. Instrum. Eng. 1211, 273-283 (1990).

15. M. Fukui, J. Tanida, and Y. Ichioka, "Flexible-structured computation based on optical array logic," Appl. Opt. 29, 1604-1609 (1990).

16. A. W. Lohmann, "Matched filtering with self-luminous objects," Appl. Opt. 7, 561-563 (1968).

17. E. G. Peak, A. V. Lehmen, J. R. Wullert II, and R. Martin, "Compact and robust incoherent holographic correlator using a surface-emitting laser-diode array," Opt. Lett. 16, 937-939 (1991).

18. I. Hayashi, "Optoelectronic integration (OEIC): the key to optical computing," in Optical Computing '88, P. Chavel, J. W. Goodman, and G. Roblin, eds., Proc. Soc. Photo-Opt. Instrum. Eng. 963, 10-14 (1989).

19. J. Jahns and A. Huang, "Planar integration of free-space optical components," Appl. Opt. 28, 1602-1605 (1989). 